\title{
Numerical Simulation on Breakup of Droplets from Vibrating Liquid Films
}

\author{
Yusuke Ohta $^{1}$, Suguru Shiratori ${ }^{1}$, Itsuhei Kohri' ${ }^{1}$, Susumu Degawa1 ${ }^{1}$, Koichi Nishibe', Akemi Ito ${ }^{1}$, \\ Nishimura Akinori², Hideaki Nagano', Kenjiro Shimano ${ }^{1}$ \\ ${ }^{1}$ Tokyo City University \\ 1-28-2 Tamazutsumi, Setagaya-ku, Tokyo 158-0087, Japan \\ ${ }^{2}$ Nabtesco Automotive Corporation \\ 2931-18, Urago-Cho 5-chome, Yokosuka, Kanagawa, 237-0062, Japan
}

\section{Extended Abstract}

A breakup of the droplets from a liquid film on the vibrating plate is investigated numerically by the two-dimensional Volume-of-Fluid method. In many industrial fluid machineries, such as internal combustion engines, compressors, or turbines, lubricant oil often unexpectedly get into an air-flow passage, and it may breakup to small droplets due to some sort of physical phenomena. If the atomized oil droplets adhere on the rubber component, which is installed at the downstream, the rubber suffers serious deteriorations. The understanding and control of such kind of atomization is highly required in order to avoid, suppress, or control the atomization. One of the possible factors in the atomization, to which the present work confines the investigation, is a vibration of a reed valve on which the oil film is adhered. Due to self-excited or forced vibration of the plate, the free surface of the liquid film adhered thereon may become unstable so that the droplets breakup from the film. As the stability of the vibrating liquid film, the so-called Faraday instability [1], by which the nonlinear standing waves are generated on the surface of the film, has been widely investigated in the previous researches. Although the detailed characteristics of the standing waves have been clarified, whether if the disturbed surface breakups to the droplets has not been investigated. The present work is addressed to clarify whether or not the vibrating liquid film breaks up to the droplets. Also, the occurrence conditions and the mechanism for the breakups are intended to be clarified. In order to achieve the above aims, the numerical simulations for the time-dependent multiphase flow were performed by means of the Volumeof-Fluid method. The initially flat liquid film was placed on the solid wall, which was moved according to the prescribed sinusoidal function. A series of the simulation was carried out with varying the frequency of the vibration, the surface tension and the viscosity of the liquid. In the result, the liquid film became to be unstable and it broke up to the small droplets, when the given inertia was stronger than certain threshold. Since this threshold was found to be dependent also on the viscosity and the surface tension of the liquid, the results whether the film broke up to the droplets were mapped onto the plane of the Reynolds number and the Weber number. From this map the occurrence condition of the breakup was estimated, and compared with the critical conditions for the Rayleigh-Taylor instability [2]. For the case where the film broke up, the diameters of the broken droplets were quantified and its dependence on the intensity of the vibration was summarized. In order to understand the mechanism of the breakups, the discretized Navier-Stokes equations were decomposed, then the viscous and the surface tension terms were separately evaluated for the time range near the breakups. The results obtained from this work is expected to contribute on the establishment of the guidelines for avoiding or suppressing the atomization.

\section{References}

[1] W. S. Edwards and S. Fauve, "Parametrically excited quasicrystal line surface waves," Phys. Rev. E, vol. 47, no. 2, pp. 788-791, 1993.

[2] Elad Sterman-Cohen, "Rayleigh-Taylor instability in thin liquid films subjected to harmonic vibration," Phys. Fluids, vol. 29, 2017. 